TENDENCIAS

Revista de la Facultad de Ciencias Económicas y Administrativas. Universidad de Nariño

Vol. XV. No. 1 - 1er. Semestre 2014,

Enero-Junio - Páginas 63-77

\title{
ALCANCES MOTIVACIONALES DE LOS ESTUDIANTES DEL PROGRAMA DE ADMINISTRACIÓN DE EMPRESAS DE LA UNIVERSIDAD DE NARIÑO, EN LAS ORGANIZACIONES
}

\author{
Por: Oscar Hernando Benavides Paz ${ }^{1}$
}

\section{RESUMEN}

La investigación asume una temática no explorada aún en la Universidad de Nariño, tiene relación directa con el futuro profesional de quienes culminan sus estudios. El estudio aborda la motivación en los estudiantes desde su desarrollo profesional y empresarial, con la finalidad de medir el impacto en cada uno de éstos ámbitos. Como principal resultado del estudio, ésta medición podrá convertirse en un factor de evaluación del Programa de Administración de Empresas, que se materialice en un currículo con seguimiento y retroalimentación continua para cumplir con el objetivo fundamental de lograr que los jóvenes encuentren posibilidades de potenciar sus propias motivaciones pensando en términos de logros, triunfos y éxitos.

Palabras clave: Motivación, Necesidad de Logro, Necesidad de Afiliación, Necesidad de Poder, Desarrollo empresarial.

Clasificación JEL: M12.

1. Profesor Asociado adscrito al Departamento de Administración de Empresas y Finanzas de la FACEA - Universidad de Nariño. Correo electrónico: oscarhbp@gmail.com

Fecha de recepción: 13 de septiembre de 2013 - Fecha de aprobación definitiva: 18 de marzo de 2014 


\title{
SCOPE MOTIVATIONAL PROGRAM STUDENT BUSINESS ADMINISTRATION UNIVERSITY NARIÑO, IN ORGANIZATIONS
}

\author{
By: Oscar Hernando Benavides Paz
}

\begin{abstract}
This research is related to a topic not yet explored in the University of Nariño and is directly related to the professional future of those who complete their studies. The research addresses the motivation in students from professional and business development, in order to measure the impact in each of these areas. This measurement will allow it to become factor and program evaluation to materialize in a curriculum which must be continuous monitoring and feedback to meet the ultimate goal of getting young people are likely to enhance their own motivations.
\end{abstract}

Keywords: Motivation Need for Achievement, Need for Affiliation, Need for Power, Business Development.

JEL Classification: M12. 


\section{JUSTIFICACIÓN}

La universidad es un centro de reflexión y crítica social permanente. Más allá de la acción perceptible a nivel de los mercados y de las generaciones, deben estar presentes los valores y las fuerzas internas o externas que dirigen el comportamiento orientado a los objetivos personales y organizacionales, que se denomina motivación, cuyo origen se remonta a los primeros hombres, para quienes la supervivencia constituía un motivador fundamental.

En todos los ámbitos de la existencia humana interviene la motivación como mecanismo para lograr determinados objetivos y alcanzar determinadas metas, representa un fenómeno humano universal de gran trascendencia para los individuos y la sociedad, para que puedan desarrollar sus talentos, habilidades, destrezas y sobre todo, la satisfacción personal de sí mismos, el cual repercutirá en su trabajo, familia y entorno.

En cada etapa de la vida, y a medida que se evoluciona y se alcanzan metas, las necesidades pueden ir cambiando, pero siempre producirán el impulso de generar un comportamiento, y un esfuerzo para satisfacer esas necesidades. Hoy, para las organizaciones, los académicos y los investigadores conocer cuáles son las motivaciones de un empleado, se ha convertido en un interrogante fundamental y en una preocupación constante en la medida en que de ésta dependen en buena parte, el éxito de un objetivo empresarial.

\section{MARCO TEÓRICO Y CONCEPTUAL}

El interés por la motivación en las organizaciones se origina con el impacto de la Revolución Industrial, que produjo cambios en el sistema de producción y la consideración del trabajador como parte de ese proceso. La motivación representa el proceso que rige las preferencias entre las distintas formas de actividad voluntaria, atribuyendo al sujeto la posibilidad de elegir entre varias conductas. Maslow (1954) define la motivación como un proceso persistente, que nunca termina, fluctúa y es compleja, característica universal de cualquier situación de un individuo. Para Claver et al (1996:299), la motivación es un proceso en el que la pasividad no tiene lugar.

En la empresa, la motivación es considerada como la acción encaminada a impulsar el comportamiento de otras personas en una dirección que se estima conveniente, en este sentido se dice que cuando una persona esta motivada en el trabajo trata de poner todo su empeño y se esfuerza por conseguir y cumplir los objetivos de la organización.

Entre las teorías o modelos más destacados que han intentado explicar la Motivación Humana se pueden mencionar 8: Teoría de la jerarquía de las necesidades de Maslow; Teoría del factor dual de Herzberg; Teoría de los tres factores de MacClelland; Teoría X y Teoría Y de McGregor; Teoría de las Expectativas; Teoría ERC de Alderfer; Teoría de la Fijación de Metas de Edwin Locke y la Teoría de la Equidad de Stancey Adams.

Robbins y Judge (2009), consideran que entre estas teorías mencionadas, la de las necesidades de McClelland, es la que tiene mayor aceptación en el ámbito de 
las investigaciones, se apoya en las teorías de Max Weber, quien sostiene que el desarrollo de los países industrializados se debía a factores culturales, entre los cuales destacaba la ética protestante.

David McClelland (1917: 98) es conocido principalmente por su trabajo desarrollado en el área de la motivación, pero, también realizó extensos estudios de la personalidad y de la conciencia. Lideró trabajos sobre pensamiento motivacional, desarrolló las bases de la teoría y de los modelos sobre logros y alcances motivacionales y promocionó mejoras en los métodos de valoración de los empleados.

MacClelland (1989), enfoca su teoría básicamente hacia tres tipos de motivación identificadas en su libro editado en 1988 "Human Motivation" como la Necesidad de Logro (n-LOG), Necesidad de Poder (n-POD) y Necesidad de Asociación (n-ASO).

Motivación de Logro. El impulso que mueve al individuo a sobresalir en correspondencia a un conjunto de patrones o modelos, dirige al sujeto a llegar lejos y tener éxito, a desarrollar las tareas de la mejor manera posible y esforzarse en ellas, a conseguir una meta basándose en la excelencia (McClelland, 1989; Robbins y Judge, 2009 y Naranjo, 2009). Este último define que los sujetos con un alto nivel de motivación al logro presentan características como: el gusto por las situaciones en las que pueden tomar personalmente la responsabilidad de encontrar la solución a los problemas, tienden a fijarse metas moderadas y a tomar riesgos calculados, deseo de una retroalimentación concreta acerca de qué tan adecuadamente se está desempeñando y se distinguen por intentar hacer bien las cosas, tener éxito, incluso por encima de las recompensas.

Motivación de Poder. Necesidad de influir sobre el pensamiento y las acciones de otras personas y grupos, y obtener reconocimiento por parte de ellas. Lleva al individuo a querer influir sobre la conducta de los demás (McClelland, 1989; Robbins y Judge, 2009).

Los individuos que poseen un alto nivel de motivación de poder emplean mayor tiempo y dedicación en "cómo obtener y ejercer el poder y la autoridad, necesitan ganar argumentos, persuadir a otros, hacer que sus criterios prevalezcan y se sienten incómodas si no poseen ciertas cuotas de autoridad” (Naranjo, 2009:160).

Motivación de Afiliación: Deseo de obtener la aceptación de los demás así como establecer relaciones cercanas con otros, por lo que dirige la conducta de la persona hacia el establecimiento de relaciones estrechas con los otros, no se sienten cómodos con el trabajo individual y les agrada trabajar en grupo y ayudar a otra gente. Quienes tienen un alto nivel de motivación de afiliación tienden a dedicar tiempo y esfuerzo en compartir momentos de calidad con otras personas y se preocupan cuando estas relaciones se ven deterioradas o presentan dificultades (McClelland, 1989; Naranjo, 2009; Robbins y Judge, 2009).

McClelland citado por Kreitner \& Kinicki (1997), afirmó que algunas personas exhiben fuertes sesgos hacia una particular necesidad motivacional o mezcla de necesidades que afectan su comportamiento y su estilo gerencial de trabajo. McClelland sugirió que una fuerte n-ASO socava la objetividad del gerente, por su necesidad de ser agradable, lo cual afecta su capacidad de tomar decisiones. Una fuerte n-POD producirá un trabajo ético y compromiso para la organización, hace 
que las personas se sientan atraídas por el rol del líder, sin embargo, no posee la flexibilidad requerida ni un "don de gentes". Las personas con n-LOG son los mejores líderes, aunque puede existir una tendencia a demandar mucho de su personal "staff" bajo la creencia de que dicho personal son todos como él, altamente motivados por el logro y los altos resultados, cosa que no siempre se manifiesta en el personal "staff".

En definitiva, esta teoría plantea que todos los individuos son diferentes y tienen intereses distintos; de ahí la importancia de conocer a fondo a los funcionarios de la empresa, y saber cuáles son las estrategias de motivación a usar con cada uno de ellos, porque algunos tendrán una tendencia más marcada hacia el logro, otros hacia el poder, la afiliación o a la combinación de estas.

\section{OBJETIVO GENERAL}

Medir los alcances motivacionales de los estudiantes del Programa de Administración de Empresas, de la Universidad de Nariño, en las organizaciones.

\section{OBJETIVOS ESPECÍFICOS}

- Seleccionar la teoría motivacional base de la investigación.

- Ponderar factores, variables e indicadores para la construcción de los instrumentos necesarios que permita la obtención de la información.

- Aplicar la encuesta para la recolección de la información documental, y medir la confiabilidad de la misma.

- Identificar las necesidades de motivación en los estudiantes.

- Analizar y presentar los resultados de la investigación.

\section{PLANTEAMIENTO DE LA INVESTIGACIÓN}

Se desarrollan los siguientes procesos: Construcción de una base de datos de estudiantes de la Universidad de Nariño del programa de Administración de empresas; elaboración de la encuesta y aplicación de la misma a estudiantes; validación de la información; tabulación de los resultados y presentación del producto Final de la investigación

\section{REVISIÓN DOCUMENTAL}

Para iniciar la investigación, y teniendo en cuenta la existencia de varias teorías o modelos que han intentado explicar la Motivación Humana, se identifican los estudios del Comportamiento Organizacional sobre la Motivación, los cuales tienen como finalidad, dar una respuesta a la pregunta: ¿Por qué trabaja la gente?, y para contestarla, la investigación se centra en la Teoría de las Necesidades de David McClelland, quien contribuyó a la comprensión de la Motivación identificando tres tipos de necesidades básicas de Motivación, de especial importancia para la administración: necesidad de poder, necesidad de asociación y necesidad de logro. 


\section{RECOLECCIÓN DE INFORMACIÓN}

Se diseñó un cuestionario de autoevaluación, con base en el modelo de la teoría de David McClelland, el cual consta de 15 preguntas, distribuidas en tres factores, cada uno con 5 ítems: Necesidad de Logro (n- LOG), Necesidad de Poder (n-POD), y Necesidad de Asociación (n-ASO).

Este cuestionario se aplicó a 221 estudiantes del programa de Administración de Empresas de la Universidad de Nariño, semestres: 2, 3, 4, 6, 8 y 10. Para evitar el sesgo que se puede presentar cuando se realiza una autoevaluación, el cuestionario fue validado posteriormente por un compañero de estudios, obteniendo dos calificaciones por cada cuestionario. La encuesta se ponderó utilizando la escala de Liker de 1 a 5 , siendo 1 el puntaje más bajo y 5 el puntaje más alto.

Cuadro 1. Logro, Poder y Afiliación según Evaluación

\begin{tabular}{|c|c|c|c|c|c|c|}
\hline \multirow{2}{*}{ Variables } & \multicolumn{2}{|c|}{ Logro } & \multicolumn{2}{c|}{ Poder } & \multicolumn{2}{c|}{ Afiliación } \\
\cline { 2 - 7 } & Hombres & Mujeres & Hombres & Mujeres & Hombres & Mujeres \\
\hline Autoevaluación & $\mathbf{4 , 3 2}$ & $\mathbf{4 , 3 0}$ & $\mathbf{3 , 8 2}$ & $\mathbf{3 , 7 0}$ & $\mathbf{3 , 6 8}$ & $\mathbf{3 , 7 1}$ \\
\hline Coevaluación & $\mathbf{4 , 2 3}$ & $\mathbf{4 , 3 7}$ & $\mathbf{3 , 7 7}$ & $\mathbf{3 , 7 3}$ & $\mathbf{3 , 7 4}$ & $\mathbf{3 , 7 9}$ \\
\hline
\end{tabular}

Fuente: Esta investigación. Pasto, 2011

Como se observa en el Cuadro 1, las diferencias que se presentan en los resultados de la evaluación y la coevaluación son poco significativas.

\section{TRATAMIENTO DE LA INFORMACIÓN}

La información recogida se somete a un tratamiento estadístico utilizando el Análisis Exploratorio, para identificar las principales características de los datos, los cuales se representan en un número reducido de gráficos que permiten medir una o más variables en la población encuestada.

Para describir el conjunto de datos se inicia con un análisis individual de las distintas variables: Género, Estrato, Edad y Ciclos. Posteriormente se estudia la relación entre ellas.

\section{ANÁLISIS INDIVIDUAL DE VARIABLES}

\section{Necesidades de Logro, Poder y Afiliación por Género}

De los estudiantes encuestados el 44,34\% corresponde a género masculino y el $55,66 \%$ femenino.

En el Gráfico 1, se observa en la población estudiada un mayor puntaje obtenido por las mujeres en la necesidad de Logro y Afiliación, y los hombres las superan, en la necesidad de Poder.

En Logro las mujeres obtienen un 4,34 y los hombres un 4.27.

En Afiliación las mujeres un 3,75 y los hombres un 3,71.

En Poder los hombres un 3,79 y las mujeres un 3,72. 


\section{Gráfico 1. Logro, Poder y Afiliación por Género}

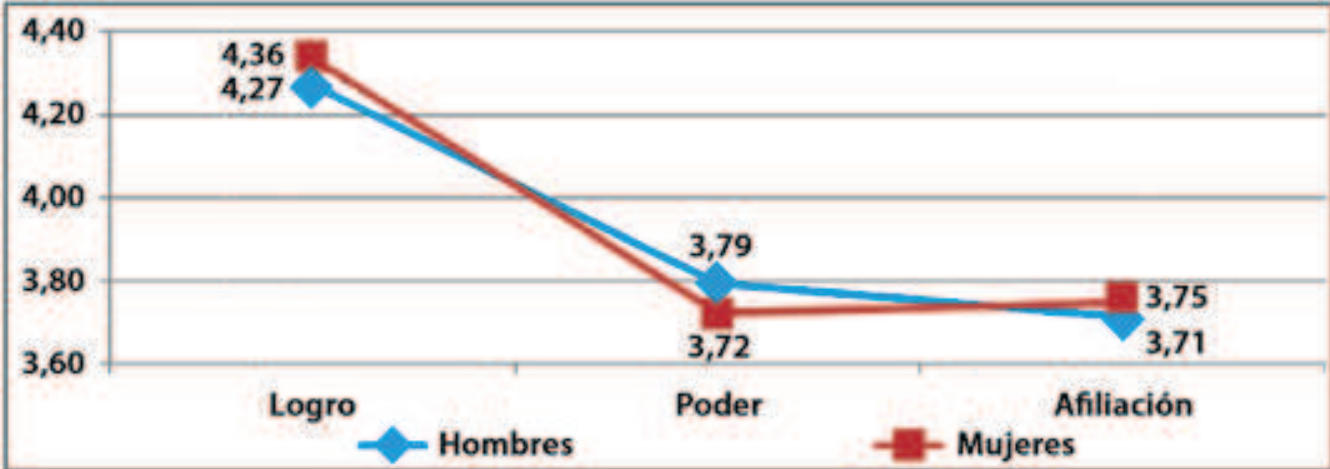

Fuente: Esta investigación. Pasto, 2011

\section{Necesidades de Logro, Poder y Afiliación por Estrato}

De la población encuestada, el 95,48\% pertenece a los estratos 1, 2 y 3 y el $4,52 \%$ corresponde a los estratos 4 y 5 .

La tendencia de las necesidades de Logro, Poder y Afiliación es en aumento, a medida que sube el estrato. Tal como se puede apreciar en el Gráfico 2.

En Logro estrato 1: 4.32 y estrato 5: 4,55.

En Poder estrato 1: 3,66 y estrato 5: 4.65.

En Afiliación estrato 1: 3,68 y estrato 5: 3,95.

\section{Gráfico 2. Logro, Poder y Afiliación por Estrato}

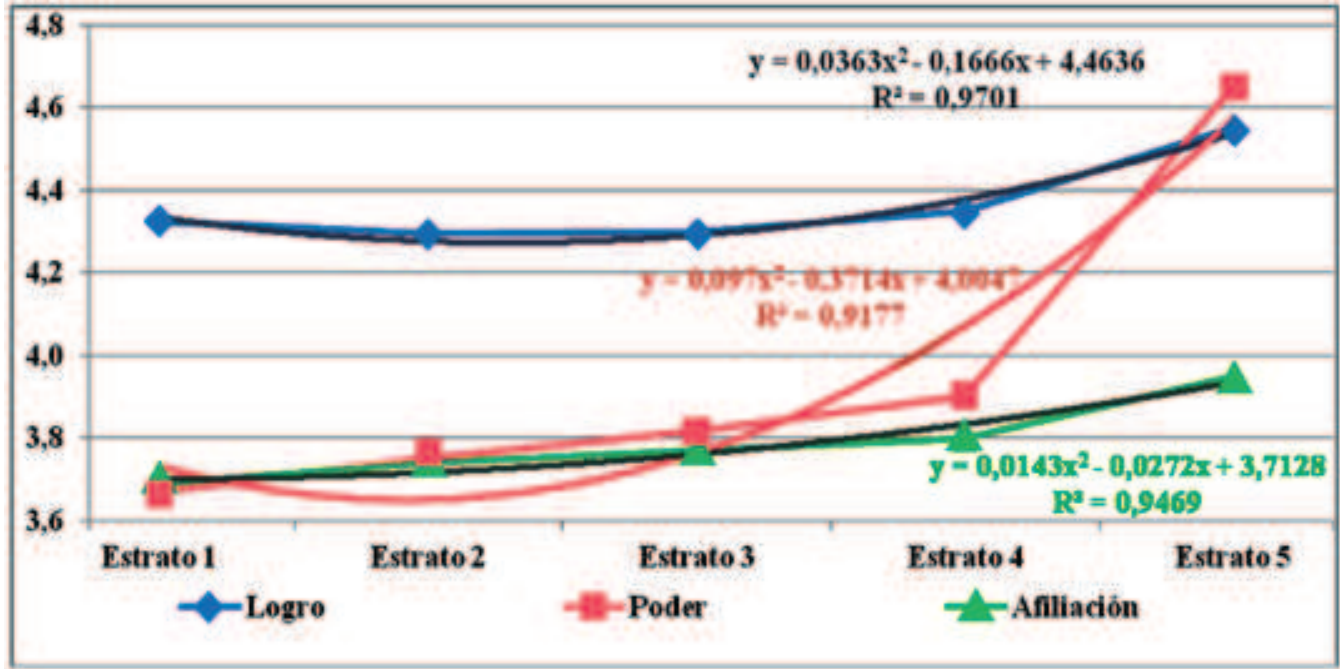

Fuente: Esta investigación. Pasto, 2011 
Este comportamiento en las variables de Logro, Poder y Afiliación como se muestra en el Gráfico 2, se asemeja a un polinomio de segundo grado, en el cual mediante ecuaciones, en un futuro se podría predecir el puntaje indicando únicamente el estrato del individuo.

$$
\begin{aligned}
& Y(\text { Logro })=0,0363 x^{2}-0,1666 x+4,4636 ; R^{2}=0,9701 \\
& Y(\text { Poder })=0,097 x^{2}-0,3714 x+4,0047 ; R^{2}=0,9177 \\
& Y(\text { Afiliación })=0,0143 x^{2}-0,0272 x+3,7128 ; R^{2}=0,9469
\end{aligned}
$$

\section{Necesidades de Logro, Poder y Afiliación por Edad}

En la población encuestada la edad oscila entre 17 y 33 años de edad. Se observan dos grupos: el primero, un rango "normal" abarca edades entre 17 y 24 años, con un porcentaje de $84,62 \%$, y el segundo grupo un rango "tardío", con edades entre 25 y 33 años, representa el 15,38\%. La edad promedio es de 21,52 años, con una desviación estándar 2,99.

Las Necesidades de Logro aumentan a medida que se incrementa la edad. En un rango de edad normal el puntaje es de 4,31 y en un rango de edad tardío es de 4,50. En las Necesidades de Poder la tendencia es a disminuir. Para un rango normal el puntaje es de 4,04 y para un rango tardío es de 3,40. En las Necesidades de Afiliación la tendencia aumenta con la edad. Pasa de un puntaje, en un rango normal de 3,90 a un puntaje de 4,00 en un rango de edad tardío (Gráfico 3).

\section{Gráfico 3. Logro, Poder y Afiliación por Edad}

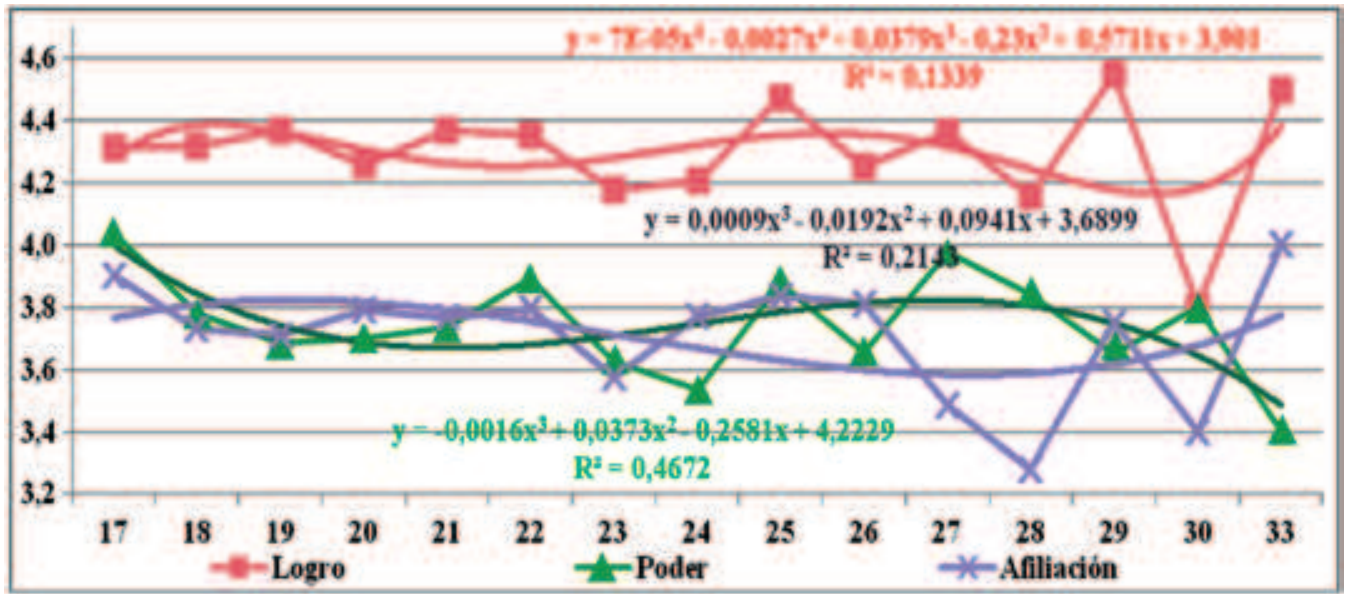

Fuente: Esta investigación. Pasto, 2011

Con la utilización de ecuaciones de polinomios de orden 5 de 3 , se puede predecir las necesidades de Logro, Poder y Afiliación indicando únicamente la variable edad. 0,1339 


$$
\begin{aligned}
& Y(\text { Poder })=-0,0016 x^{3}+0,0373 x^{2}-0,2581 x+4,2229 ; R^{2}=0,4672 \\
& Y(\text { Afiliación })=0,0009 x^{3}-0,0192 x^{2}+0,0941 x+3,6899 ; R^{2}=0,2143
\end{aligned}
$$

\section{Necesidades de Logro, Poder y Afiliación por Ciclos}

Del total de 221 estudiantes encuestados el 52,04\% corresponde al Ciclo Básico (semestres: 2, 3, 4 y 6) y el 47,96\% al Ciclo Profesional (semestres 8 y 10). El puntaje que se obtiene en Necesidades de Logro es más alto en el ciclo profesional 4,35, que en el ciclo básico 4,27. En las Necesidades de Poder no se presentan diferencias significativas entre los dos ciclos. En las Necesidades de Afiliación se obtiene un puntaje más alto en el ciclo básico 3,81, frente al ciclo profesional 3,61 (Gráfico 4).

\section{Gráfico No 4. Logro, Poder y Afiliación por Ciclos}

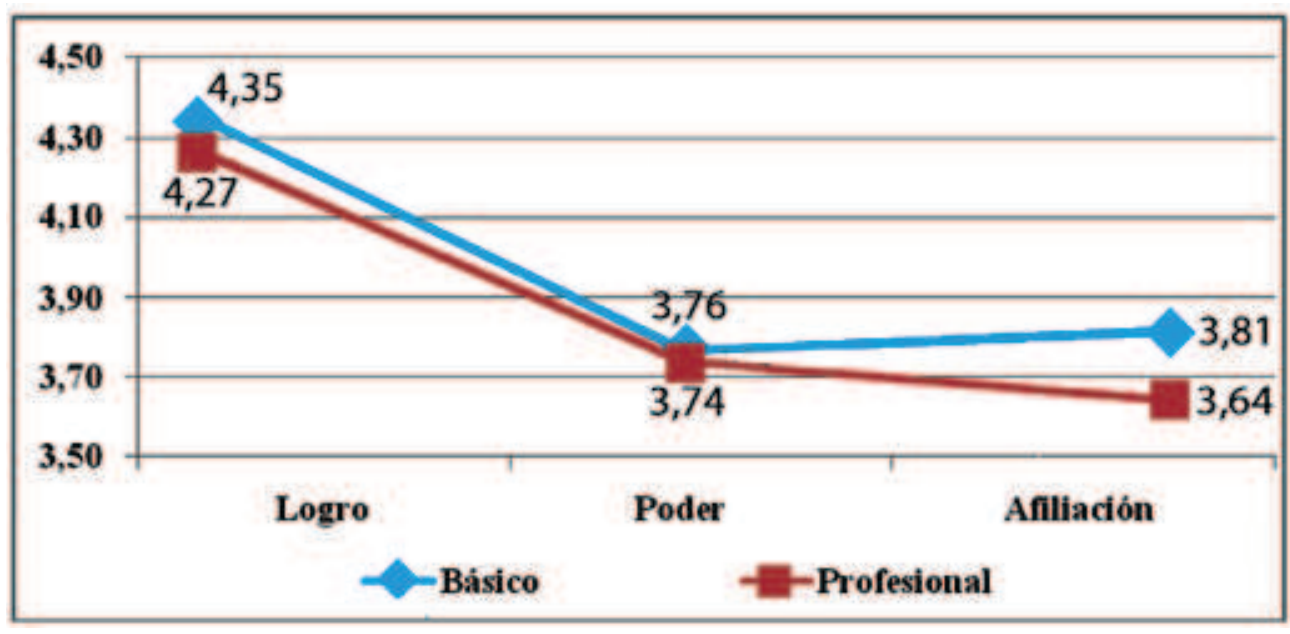

Fuente: Esta investigación. Pasto, 2011

\section{RELACIÓN ENTRE VARIABLES}

\section{Necesidades de Logro, Poder y Afiliación por Estrato y Género}

En las necesidades de Logro se observa un aumento en los hombres a medida que sube el estrato, de 4,25 en el estrato 1 a 4,55 en el estrato 5, a diferencia de las mujeres donde se observa que la participación disminuye de 4,38 en el estrato 1 a 4,30 en el estrato 4 .

La necesidad de Poder en los hombres aumenta de 3,64 en el estrato 1 a 4,65 en el estrato 5, en las mujeres igualmente aumenta de 3,69 en el estrato 1 a 3,74 en el estrato 4. 
La necesidad de Afiliación en los hombres aumenta al pasar de 3,65 en el estrato 1 a 3,95 en el estrato 5, y en las mujeres aumenta también levemente de 3,71 en el estrato 1 a 3,74 en el estrato 4 (Cuadro 2).

Cuadro 2. Logro, Poder y Afiliación por Estrato y Género

\begin{tabular}{|l|c|c|c|c|c|c|}
\hline \multirow{2}{*}{ Estrato } & \multicolumn{2}{|c|}{ Logro } & \multicolumn{2}{c|}{ Poder } & \multicolumn{2}{c|}{ Afiliación } \\
\cline { 2 - 7 } & Hombres & Mujeres & Hombres & Mujeres & Hombres & Mujeres \\
\hline Estrato 1 & 4,25 & 4,38 & 3,64 & 3,69 & 3,65 & 3,71 \\
\hline Estrato 2 & 4,33 & 4,27 & 3,88 & 3,67 & 3,74 & 3,74 \\
\hline Estrato 3 & 4,21 & 4,42 & 3,76 & 3,90 & 3,71 & 3,85 \\
\hline Estrato 4 & 4,43 & 4,30 & 4,17 & 3,74 & 3,90 & 3,74 \\
\hline Estrato 5 & 4,55 & - & 4,65 & - & 3,95 & - \\
\hline
\end{tabular}

Fuente: Esta investigación. Pasto, 2011

\section{Necesidades de Logro, Poder y Afiliación por Género y Ciclos}

En las necesidades de Logro las mujeres presentan puntajes más altos que los hombres en los dos ciclos básico y profesional. En las necesidades de poder se observa que las mujeres obtienen puntajes más altos en el ciclo básico (semestres 2, 3,4 y 6) y menores que los hombres en el ciclo profesional (semestres 8 y 10). En las necesidades de Afiliación los resultados se alternan, en unos semestres obtienen mejores puntajes los hombres y en otros las mujeres (Cuadro 3).

Cuadro 3. Logro, Poder y Afiliación por Ciclos y Género

\begin{tabular}{|c|c|c|c|c|c|c|}
\hline \multirow{2}{*}{ Semestres } & \multicolumn{2}{|c|}{ Logro } & \multicolumn{2}{c|}{ Poder } & \multicolumn{2}{c|}{ Afiliación } \\
\cline { 2 - 7 } & Hombres & Mujeres & Hombres & Mujeres & Hombres & Mujeres \\
\hline 2 & 4,36 & 4,51 & 3,66 & 3,96 & 3,50 & 3,87 \\
\hline 3 & 4,38 & 4,47 & 3,73 & 3,84 & 3,88 & 3,72 \\
\hline 4 & 4,13 & 4,14 & 3,67 & 3,48 & 3,69 & 3,70 \\
\hline 6 & 4,34 & 4,51 & 3,91 & 3,82 & 3,99 & 4,09 \\
\hline 8 & 4,13 & 4,26 & 3,83 & 3,68 & 3,49 & 3,74 \\
\hline 10 & 4,26 & 4,30 & 3,86 & 3,68 & 3,68 & 3,60 \\
\hline
\end{tabular}

Fuente: Esta investigación. Pasto, 2011

En resumen como se muestra en el Gráfico 5, se pueden observar los puntajes obtenidos por el total de la población encuestada, 221 estudiantes, en las tres variables: Logro 4.31, Poder 3.75 y Afiliación 3.73. 


\section{Gráfico 5. Logro, Poder y Afiliación}

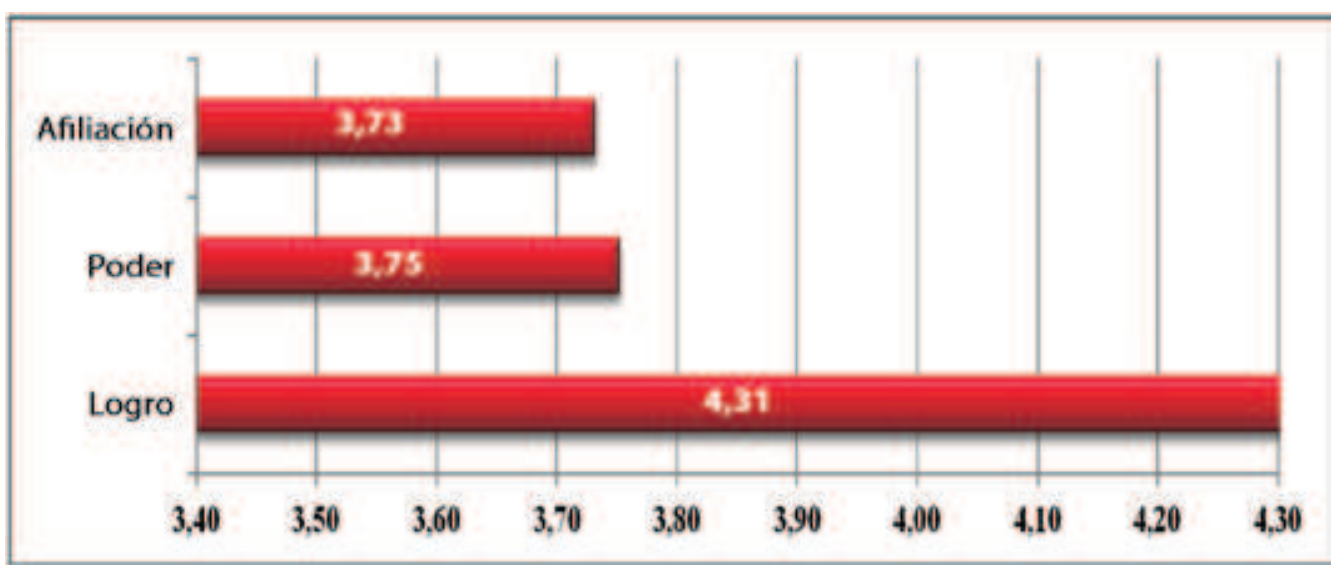

Fuente: Esta investigación. Pasto, 2011

\section{PRUEBAS DE HIPÓTESIS}

Se hace necesario este procedimiento, con el fin de extraer conclusiones que permitan aceptar o rechazar las hipótesis planteadas.

HIPÓTESIS 1. En los estudiantes de Administración de Empresas de la Universidad de Nariño, el Género no influye en las necesidades de Logro, Poder y Afiliación (Cuadro 4).

Hipótesis Nula. El género en los estudiantes de Administración de Empresas de la Universidad de Nariño influye en las necesidades de Logro, Poder y Afiliación.

Hipótesis nula: $\quad$ mu1 $=$ mu2

Hipótesis alterna: $\mathrm{mu} 1 \neq \mathrm{mu} 2$

Cuadro 4. Necesidades de Logro, Poder y Afiliación por Género

\begin{tabular}{|c|c|c|c|c|c|c|}
\hline \multirow{2}{*}{ Género } & \multicolumn{3}{|c|}{ Hombres } & \multicolumn{3}{|c|}{ Mujeres } \\
\hline & Logro & Poder & Afiliación & Logro & Poder & Afiliación \\
\hline Puntaje & 4,27 & 3,79 & 3,71 & 4,34 & 3,72 & 3,75 \\
\hline Desviación Estándar & 0,448 & 0,609 & 0,598 & 0,501 & 0,611 & 0,556 \\
\hline Observaciones & 196 & 196 & 196 & 246 & 246 & 246 \\
\hline Estadístico Z Calculado & $-1,529$ & 1,198 & $-0,7266$ & & & \\
\hline Valor-P & 0,1263 & 0,2308 & 0,4675 & & & \\
\hline Intervalo de Confianza & {$[-0,1597,0,01974]$} & {$[-0,04449,0,1845]$} & {$[-0,1479,0,0679]$} & & & \\
\hline Hipótesis Nula Alfa $=0,05$ & No Rechazar & No Rechazar & No Rechazar & & & \\
\hline
\end{tabular}

Fuente: Esta investigación. Pasto, 2011 
Se observa en el análisis de la información obtenida que el género no influye en las necesidades de Logro, Poder y Afiliación. Por lo tanto la Hipótesis es aceptada.

HIPÓTESIS 2. El estrato no influye en las necesidades de Logro, Poder y Afiliación de los estudiantes de Administración de la Universidad de Nariño.

Hipótesis Nula. El estrato es una variable que influye en las necesidades de Logro, Poder y Afiliación de los estudiantes de la Universidad de Nariño.

Según la información obtenida y que se observa en el Cuadro 5 esta Hipótesis es aceptada.

Cuadro 5. Necesidades de Logro, Poder y Afiliación según Estrato

\begin{tabular}{|c|c|c|c|c|c|c|}
\hline \multirow{2}{*}{ Estrato } & \multicolumn{3}{|c|}{ Estrato Bajo } & \multicolumn{3}{|c|}{ Estrato Medio } \\
\hline & Logro & Poder & Afiliación & Logro & Poder & Afiliación \\
\hline Puntaje & 4,31 & 3,71 & 3,71 & 4,31 & 3,83 & 3,77 \\
\hline Desviación Estándar & 0,487 & 0,626 & 0,587 & 0,458 & 0,542 & 0,545 \\
\hline Observaciones & 318 & 318 & 318 & 120 & 120 & 120 \\
\hline Estadístico Z Calculado & 0,0 & $-1,854$ & $-0,9726$ & & & \\
\hline Valor-P & 1,0 & 0,06378 & 0,3308 & & & \\
\hline Intervalo de Confianza & {$[-0,1006,0,1006]$} & {$[-0,2469,0,006878]$} & {$[-0,1809,0,06092]$} & & & \\
\hline Hipótesis Nula Alfa $=0,05$ & No Rechazar & No Rechazar & No Rechazar & & & \\
\hline
\end{tabular}

HIPÓTESIS 3. La edad es una variable que no influye en las necesidades de Logro, Poder y Afiliación de los estudiantes de Administración de Empresas de la Universidad de Nariño.

Hipótesis Nula. La edad influye en las necesidades de Logro, Poder y Afiliación en los estudiantes de Administración de Empresas de la Universidad de Nariño.

La información obtenida según el Cuadro 6, demuestra que la hipótesis es aceptada.

Cuadro 6. Necesidades de Logro, Poder y Afiliación según Edad

\begin{tabular}{|c|c|c|c|c|c|c|}
\hline \multirow{2}{*}{ Edad } & \multicolumn{3}{|c|}{ Edad 1 (Apropiada) } & \multicolumn{3}{|c|}{ Edad 2 (Avanzada) } \\
\hline & Logro & Poder & Afiliación & Logro & Poder & Afiliación \\
\hline Puntaje & 4,31 & 3,75 & 3,75 & 4,28 & 3,78 & 3,60 \\
\hline Desviación Estándar & 0,474 & 0,627 & 0,583 & 0,519 & 0,472 & 0,494 \\
\hline Observaciones & 390 & 390 & 390 & 52 & 52 & 52 \\
\hline Estadístico Z Calculado & 0,4239 & $-0,3326$ & 1,772 & & & \\
\hline Valor-P & 0,6717 & 0,7395 & 0,0764 & & & \\
\hline Intervalo de Confianza & {$[-0,1087,0,1687]$} & {$[-0,2068,0,1468]$} & {$[-0,01591,0,3159]$} & & & \\
\hline Hipótesis Nula Alfa = 0,05 & No Rechazar & No Rechazar & No Rechazar & & & \\
\hline
\end{tabular}

Fuente: Esta investigación. Pasto, 2011 
HIPÓTESIS 4. El ciclo de estudios no influye en las Necesidades de Logro, Poder y Afiliación de los estudiantes de Administración de la Universidad de Nariño.

Hipótesis Nula. El ciclo de estudios influye en las necesidades de Logro, Poder y Afiliación.

La información obtenida en el Cuadro 7 demuestra que la hipótesis es aceptada.

Cuadro 7. Necesidades de Logro, Poder y Afiliación según Ciclos

\begin{tabular}{|c|c|c|c|c|c|c|}
\hline \multirow{2}{*}{ Ciclo } & \multicolumn{3}{|c|}{ Ciclo Básico } & \multicolumn{3}{|c|}{ Ciclo Profesional } \\
\hline & Logro & Poder & Afiliación & Logro & Poder & Afiliación \\
\hline Puntaje & 4,35 & 3,76 & 3,81 & 4,27 & 3,74 & 3,81 \\
\hline Desviación Estándar & 0,448 & 0,616 & 0,559 & 0,507 & 0,606 & 0,579 \\
\hline Observaciones & 230 & 230 & 230 & 212 & 212 & 212 \\
\hline Estadístico Z Calculado & 1,761 & 0,3437 & 0,0 & & & \\
\hline Valor-P & 0,07827 & 0,7311 & 1,0 & & & \\
\hline Intervalo de Confianza & {$[-0,00905,0,169]$} & {$[-0,09406,0,1341]$} & {$[-0,1061,0,1061]$} & & & \\
\hline Hipótesis Nula Alfa $=0,05$ & No Rechazar & No Rechazar & No Rechazar & & & \\
\hline
\end{tabular}

Fuente: Esta investigación. Pasto, 2011.

\section{CONCLUSIONES Y RECOMENDACIONES DE LA INVESTIGACIÓN}

Las conclusiones y recomendaciones como producto de los resultados que arroja esta investigación están dirigidos a la reflexión del programa de Administración de Empresas, de la Universidad de Nariño, en su afán de formar líderes, con una adecuada motivación, estimulando y desarrollando sus necesidades de Poder y Afiliación, propiciando que los estudiantes piensen en términos de Logros, triunfos y éxitos; con el fin de presentar a la comunidad empresarial candidatos adecuados para los puestos que lo demanden.

La investigación muestra que los estudiantes de Administración de Empresas de la Universidad de Nariño presentan un puntaje de 4,31 en la necesidad de Logro. Sin embargo, una necesidad alta de Logro no necesariamente lleva a ser un buen gerente, sobre todo en las grandes organizaciones. La persona con una alta necesidad de logro esta interesada en qué tan bien se desempeña personalmente y no en influenciar a sus colaboradores para que hagan bien su trabajo.

En la necesidad de Poder los estudiantes presentan un puntaje de 3,75 y 3,73 en la necesidad de Afiliación. Estas dos necesidades de afiliación y de poder tienden a estar estrechamente relacionadas con el éxito gerencial. Los mejores gerentes tienen una alta necesidad de poder y una baja necesidad de afiliación. 
Una alta motivación de poder podría ser un requisito de la eficacia gerencial.

La información obtenida permitió realizar el contraste de hipótesis que demuestra que los estudiantes de Administración de la Universidad de Nariño, están preparados para asumir grandes retos en las empresas a nivel regional y nacional.

No todas las motivaciones de los individuos parten de necesidades congruentes con las metas organizacionales. Por lo tanto, para considerar que a un trabajador le motiva su trabajo, las necesidades del individuo deberán ser compatibles con las metas de la organización.

Las diferentes teorías sobre motivación no siempre son enteramente aplicables a todas las realidades. Depende de la cultura, las costumbres, los valores, las situaciones sociales, económicas y otros factores, que condicionan el modo de pensar y actuar de los individuos, situaciones que inciden en los estudios que se realizan en relación con la motivación.

Considerando el proceso de motivación, es posible afirmar que ésta no se puede dominar, por diferentes razones: no todas las personas tienen los mismos deseos; las personas no se comportan siempre igual, aunque tengan las mismas necesidades; al final, los resultados no acompañan necesariamente las expectativas del motivador y del subordinado y las reacciones de las personas son imprevisibles logrando o no, sus deseos.

Una organización esta conformada por un conjunto de personas cuyo desempeño está directamente relacionado con el rendimiento de sus empleados y directivos, demostrado así, la influencia que tiene la motivación sobre la conducta, el desempeño laboral y la calidad de productos y servicios. El papel que juega el factor humano y su motivación en el seno de una organización es la garantía para el cumplimiento de los objetivos y metas organizacionales. 


\section{REFERENCIAS}

CLAVER, E.; GASCÓ, J.L. y LLOPIS, J. (1996). Los recursos humanos en la empresa: un enfoque directivo, Editorial Civitas, Madrid.

HELLRIEGEL, Don, JACKSON, Susana y SLOCUM, Jhon (2005). Administración: un enfoque basado en competencias. Editorial International Thompson, Bogotá, novena edición.

LIND, Douglas, MARCHAL, William y WATHEN, Samuel (2005). Estadística aplicada a los negocios y la economía. Editorial McGraw Hill, México, décimo segunda edición.

MASLOW, Abraham H. (1954). Motivation and personality. Harper, New York.

McCLELLAND, David C. (1961). Methods of measuring human motivation, in John W. Atkinson, ed.,The Achieving Society (Princeton, N.J.: D. Van Nostrand, 1961), pp. 41-43.

McCLELLAND, David C. (1965). Achievement motivation can be developed, Harvard Business Review 43 (November-December), p. 68.

ROBBINS, S. \& JUDGE, T. A. (2009). Comportamiento organizacional. Educación. Pearson, México.

WEIERS, Ronald M. (2006). Introducción a la estadística para negocios. International Thompson Editores, México, quinta edición. 